patients-those being denied follow up-would be getting worse care. Evidence is accumulating to support the idea that routine follow up confers little benefit to patients. Certainly, the benefit of outpatient review after one of the commonest urological procedures, transurethral resection of the prostate, has recently been questioned. ${ }^{3}$ Perkins et al found that $92 \%$ of patients were discharged at their first postoperative visit and only $2 \%$ remained under review at one year. Moreover, $90 \%$ of general practitioners and $78 \%$ of patients expressed confidence in a system without routine hospital review.

Similar questions now exist with regard to longer term follow up in other specialties. Colorectal surgeons have become increasingly pessimistic about the usefulness of routine outpatient follow up compared with opportunistic detection of early recurrence of colorectal cancer by general practitioners. ${ }^{45}$ In the only randomised study across surgical specialties that compared follow up by general practitioners and hospital outpatient departments, reoperation rates, mortality, and cost to providers were similar for the two groups. ${ }^{6}$ The cost to patients in terms of time and money was greater for the group attending hospital outpatients. Despite acknowledging that more work would result, general practitioners were in favour of immediate hospital discharge.

Two themes emerge. Firstly, these reports and others 7 highlight the fact that patients, if well informed, are good at detecting and reporting complications or clinical deterioration -and, importantly, they tend to report them to their general practitioner. The second relates to access. It is ironic that provider units that decide to provide a comprehensive outpatient follow up service are likely to have no spare capacity to respond quickly to a phone call or letter from the patient or general practitioner requesting an early unscheduled appointment.

These two areas will require attention if community follow up is to be widely adopted. At present, patients find that written information on their postoperative recovery and possible complications is inadequate for their needs. ${ }^{8}$ In addition, there is increasing recognition of the importance of access to outpatients departments and of patients' perception of access, since better access improves health care outcomes. ${ }^{9}$ With a little imagination surgeons could do much to improve access to urgently requested appointments. Both the general practitioner and patient need to know that, should an outpatient appointment be necessary, it is no more than a phone call away.

MARK EMBERTON

St Georges Hospital NHS Trust, Senior registrar in urology

London SW17 ORE

1 Waghom A, Thompson J, McKee, M. Routine surgical follow up: do surgeons agree? BMf 1995; 311:1344-5.

2 Bailey M, Boyd J. South West London Patient Care Review, 1995. South West London Acute Services Purchaser Provider Group, April 1995.

3 Perkins JMT, O'Brien TS, Hanbury DC, Cranston DW. Is follow up necessary after transurethral resection of the prostate? Br $\mathcal{G}$ Urol 1995;75:618-21.

4 Cochrane J, Williams J, Faber R, Slack W. Value of outpatient follow up after curative surgery for carcinoma of the large bowel. $B M \Im 1980 ; 280: 593-5$.

5 Wyatt J, Aitkin R. Evaluation of hospital and general practice follow-up after surgery for colorectal cancer. Brf Surg 1994;81:145.

6 Florey CDV, Yule B, Fogg A, Napier A, Orbell S, Cuschieri A. A randomised controlled trial of immediate discharge of patients to general practice. f Public Health Med 1995;16:455-64.

7 Dunn J, Bell A, Elliot T, Kernick V, Lavy J, Campbell W. Outpatient clinic review after arterial reconstruction: is it necessary? Ann $R$ Coll Surg Engl 1994;76:304-6.

8 Meredith P, Emberton M, Wood C, Smith J. Comparisons of patients needs for information on prostate surgery with printed materials provided by surgeons. Quality in Health Care 1995;4: $18-23$

9 Starfield B. Access-perceived or real, and to what? YAMA 1995;274:346-7.

\title{
Psychosocial interventions in cancer
}

\author{
Should be part of every patient's management plan
}

Growing awareness of the many psychosocial problems associated with malignant disease and its treatment has led to the development of a myriad of supportive interventions for patients and their families. These interventions range from traditional approaches such as psychotherapy to the less orthodox music therapy and aromatherapy. They may be offered formally or informally by health care professionals or by lay volunteers, including patients themselves. Some psychosocial interventions, such as counselling by specialist nurses in breast cancer units, have been incorporated into the routine care of patients with cancer. Other interventions may be provided outside hospitals by a wide variety of self help groups or national and local cancer support organisations. In addition, private practitioners offer everything from psychotherapy to therapeutic massage on a fee paying basis.

The mere existence of so many different approaches shows that the demand for this form of support is considerable. The question for patients and purchasing authorities is: what impact do these forms of psychosocial intervention have on the wellbeing of patients with cancer? Despite considerable anecdotal evidence attesting to their benefits, objective evidence of efficacy has not been compelling. ${ }^{1-3}$ Significant and non-significant results from methodologically inadequate studies purporting to evaluate psychosocial interventions have, if anything, hampered their integration into the formal care and management of patients with cancer. ${ }^{4}$ The dearth of good empirical data can be partly explained by the fact that the activities covered by the term psychosocial intervention vary widely with regard to the training and ability of therapists, their relationship with the patient, the nature and content of the intervention, the primary goals, and the predicted outcomes. ${ }^{5}$ However, a review of the literature that focuses on the four mainstream psychosocial interventions-behavioural therapy (including relaxation, biofeedback, and hypnosis); educational therapy (including training in coping skills and providing information to enhance a patient's sense of control); psychotherapy (including counselling); and support groups (which help patients to express their emotions)-shows that there is increasing evidence of efficacy. ${ }^{6-9}$

Published controlled studies have shown positive benefits. Examples include a reduction in the side effects of chemotherapy after biofeedback and relaxation therapy ${ }^{10} ;$ a significant reduction in psychological morbidity after cognitive and behavioural therapy ${ }^{8}$; improved coping skills after psychoeducational approaches ${ }^{7}$; and a reduction in pain, less mood disturbance, and fewer maladaptive coping responses after supportive group therapy. ${ }^{11}$ More contentiously and provocatively, some researchers have suggested that psychosocial interventions not only improve the quality of patients' lives but also extend their survival. ${ }^{71}$ The fact that controlled studies of psychosocial interventions show beneficial effects is remarkable, given the small numbers of patients in such studies and innumerable confounding factors influencing the 
outcomes. But modest positive effects, albeit with low statistical power, are clearly observable.

More convincing evidence for efficacy was provided recently by a meta-analysis of the effects of psychosocial interventions in adults with cancer. ${ }^{12}$ Meyer and Mark retrieved 62 studies comparing treatment and control groups for various psychosocial, behavioural, and psychoeducational interventions. They classified the outcomes of each study into five summary categories: (1) emotional adjustment, which included outcomes such as mood state, self esteem, locus of control, denial, and repression; (2) functional adjustment, which consisted of outcomes such as socialising and returning to work; (3) symptoms related to the disease or treatment, including nausea, vomiting, and pain; (4) medical outcomes, such as leucocyte activity, the response of the tumour, and the progression of disease; and a final category, (5) global outcomes, in which several areas from the four other categories were combined. The results of the meta-analysis were expressed as a unit free effect size ranging from 0 to 1 , calculated by dividing the difference in the means of the control and treatment groups by the pooled standard deviation. They showed significant beneficial effect sizes of from 0.19 to 0.28 for all the summary categories except the medical outcomes category, which at 0.17 was not significant. These positive effects are within the range expected for psychological procedures that "work"; they are, however, small, and consequently some people might question their clinical importance. Meyer and Mark point out that within medicine in general even quite modest effects can have an important impact on the outcome of treatment.

Although type II statistical errors are possible because of small effect sizes and relatively few treatment-control comparisons, no significant differences in effect size were found between the different summary categories for different types of psychosocial interventions. This is worthy of further research. We need more information about the most effective types of interventions, as well as some innovative work on different combinations that might produce even greater benefits to patients.
Meta-analysis has become an increasingly important and established method of determining benefit from medical procedures. The analysis conducted by Meyer and Mark is the first that explicitly examines the putative benefits of psychosocial interventions for patients with cancer. The results, together with the data now accumulating from larger, more methodologically sound evaluations, begin to place psychological interventions firmly on the list of requirements for good cancer care. Such interventions should no longer be seen as an optional extra but as an integral part of every patient's management plan. The delivery of medical care is becoming increasingly evidenced based; for the non-believers in psychosocial care here is that evidence.

LESLEY FALLOWFIELD

Reader in psycho-oncology

CRC Communication and Counselling Research Centre,

Department of Oncology,

University College London Medical School,

Bland Sutton Institute,

London W1P 7PL

1 Cunningham AJ. From neglect to support to coping: the evolution of psychosocial intervention for cancer patients. In: Cooper CL, ed. Stress and breast cancer. Chichester: John Wiley, 1988: 135-54.

2 Massie MJ, Holland J, Straker N. Psychotherapeutic interventions. In: Holland J, Rowland JH, eds. Handbook of psycho-oncology. New York: Oxford University Press, 1989:455-69.

3 Fallowfield LJ. Evaluation of counselling in the National Health Service. $尹 R$ Soc Med 1993;86: 429-30.

4 Martin E. Counsellors in general practice. BMF 1988;297:637-8.

5 Fallowfield LJ, Roberts R. Cancer counselling in the United Kingdom. Psychology and Health 1992;6:107-17.

6 Cunningham AJ. Group psychological therapy for cancer patients: a brief discussion of indications for its use, and the range of interventions available. Supportive Care in Cancer 1995;3:244-7.

7 Fawzy IF, Fawzy NW, Arndt LA, Pasnau RO. A critical review of psychosocial interventions in cancer care. Archives of General Psychiatry 1995;52:100-13.

8 Greer S, Moorey S, Baruch JDR, Watson M, Robertson BM, Mason A, et al. Adjuvan psychological therapy for patients with cancer: a prospective randomised trial. BMF 1992;304: 675-80. 9 Spiegel D. Commentary: psychosocial intervention in cancer. I Natl Cancer Inst 1993;85:

10 Burish TG, Jenkins RA. Effectiveness of biofeedback and relaxation training in reducing the sideeffects of cancer chemotherapy. Health Psychology 1992;11:65-78.

11 Spiegel D, Bloom JR, Kraemer HC, Gottheil E. Effect of psychosocial treatment on survival of patients with metastatic breast cancer. Lancet 1989;ii:888-91.
piss

12 Meyer TJ, Mark MM. Effects of psychosocial interventions with adult cancer patients: a metaanalysis of randomized experiments. Health Psychology 1995;14:101-8.

\section{Registering patients and paying capitation in family practice}

\section{Lessons from Canada}

It may surprise British doctors to learn that universal registration of citizens for primary care is controversial and exists almost nowhere else in the world. They might ask: "How can one take responsibility for a patient's preventive care, and for facilitating appropriate use of community services and referred care, without a defined list of patients?" Indeed, unsatisfactory answers to such questions, implying a less than adequate primary care system, have provided impetus for considering patient registration in other countries. ${ }^{1}$ We use the current controversy in Canada to explore the issue.

Currently, primary care in Canada is delivered on a fee for service basis by doctors operating privately in solo or group practices. These doctors have no contractual obligations to government payers with regard to location, the mix of services provided, or the organisation of the practice. Although some special arrangements exist to improve the geographical distribution of doctors or to experiment with capitation payment or health centre models, ${ }^{2}$ these cover less than $5 \%$ of the population.
Canada's federal and provincial governments and its doctors support the idea of changing to a system based on registration. But they have also expressed their concerns. ${ }^{3-6}$ The present debate centres on a report on the funding and reorganisation of primary care that was released for consultation this autumn by the federal, territorial, and provincial committee advising the government on health services. ${ }^{7}$ The details of the model proposed by the committee are less important than the principles: to move away from fee for service payment, register patients with a primary care organisation (which has defined contractual commitments), and pay each primary care organisation on a capitation basis. Similar proposals have come from various doctors' organisations in Canada that are interested in registration, comprehensive care, and new payment options. ${ }^{34}$ Registration has therefore become intertwined with the debate on how to pay doctors. ${ }^{8}$ The result is a guaranteed controversy.

For Canada's governments the impetus is mostly to replace a fee for service system, which encourages the provision of an ever increasing volume of services, with a more predictable 